\title{
Analysis of Multiple-duct Variable Area Perforated Tube Resonators
}

\author{
T. Kar, P. P. R. Sharma and M. L. Munjal ${ }^{\dagger}$ \\ Facility for Research in Technical Acoustics, Department of Mechanical Engineering, \\ Indian Institute of Science, Bangalore 560 012, India
}

(Received 15 July 2004; accepted 18 October 2005)

\begin{abstract}
Conical concentric tube resonators are often used in commercial automotive mufflers. These are characterised by wave-coupling phenomenon across interacting ducts. Using a one-dimensional control volume approach, a mathematical model is presented for a generalised configuration of variable area perforated tube resonators. The analysis is applied to different configurations that account for waves in the incompressible mean flow and in the acoustic coupling between the interacting ducts due to the admittance of the intervening perforates. The problem has been solved by means of the Peano-Baker's series of matrix calculus, and the transmission loss results have been outlined. The predictions have been validated against the three-dimensional finite element analysis.
\end{abstract}

${ }^{\dagger}$ Fellow of the International Institute of Acoustics and Vibration (IIAV)

\section{Nomenclature}

$a_{0}$ - speed of sound in air

$f$ - frequency $(\mathrm{Hz})$

$j$ - imaginary unit

$k_{0}$ - wavenumber

$M_{i}$ - flow Mach number in the $i$-th duct

$p_{i}-$ acoustic pressure

$S_{i}$ - cross-sectional area of the $i$-th duct

$U_{i}$ - flow velocity in the $i$-th duct

$u_{i}$ - particle velocity fluctuation over $U_{i}$

$u_{i k}^{*}$ - radial particle velocity at the interface of the $i$-th and $k$-th ducts

$Y_{0}$ - characteristic impedance of air

\section{Greek Symbols}

$\zeta_{i k}$ - perforate impedance at the interface of the $i$-th and $k$-th ducts

$\lambda$ - latent root

$\mu-$ relative performance index

$\rho_{0}-$ air density

$\rho_{i}$ - density perturbation over $\tilde{\rho}_{i}$ or $\rho_{0}$, in the $i$-th duct

$\sigma_{i k}$ - porosity of the perforated interface

\section{Subscripts}

$0-$ air

$i-i$-th duct

$i k$ - interface of the $i$-th and $k$-th ducts; the $i$-th row, $k$-th column element (of a matrix)

\section{INTRODUCTION}

A perforated tube surrounded by a uniform annular cavity forms a concentric tube resonator (CTR), which is used widely in the intake systems as well as the exhaust systems of automobiles. ${ }^{1}$ A generalised algorithm has been presented for the coefficients of the system matrix of a muffler configuration with any number of uniform-area (parallel) interacting ducts. ${ }^{2}$ The corresponding matrizant analysis, though limited to multiple coaxial ducts, has been reported elsewhere. ${ }^{3}$ The perforated tube may combine with a variable area cavity to form a variable area concentric tube resonator (VCTR). The analytical model of the plane wave propagation and the transmission loss (TL) spectrum for one such primitive model has been reported recently. ${ }^{4}$

Sound transmission in a circular duct of continuously varying area was predicted by Alfredson ${ }^{5}$ by the more primitive segmentation approach, which was in essence a segmentation of the duct into a number of subsections of uniform tubes with area discontinuity at the junction of each pair of subsections. The transmission matrix of a variable area duct carrying a compressible subsonic flow was then developed by Miles ${ }^{6}$ with the assumption of a constant mean flow for each individual segment.

A solution of the wave equation with variable coefficients for a nonuniform duct with a moving medium was provided by Dokumaci, ${ }^{7}$ making use of the matrizant method. ${ }^{8}$ Later, he presented an exact transfer matrix formulation by making use of the Riccati equation of duct impedance for plane sound wave propagation, which applies to a single duct with inhomogeneities due to the axial gradient in the ambient conditions as well as the cross-sectional area variations. ${ }^{9}$ It was later used to evaluate the performance of the acoustic elements used in the variable area CTRs. The conclusion drawn from the TL spectrum of the conical concentric tube resonators (CCTR $)^{4}$ is not entirely generic for all sorts of variable area CTRs, as an additional geometric parameter may play a role in shaping the generality into a different dimension.

Incidentally, several investigations have been reported in the literature on perforated tubes. ${ }^{10,11}$ Modification to the multiple-duct variable area CTRs in the acoustic elements may open up new options for a designer. Inclusion of a number of variable area ducts in a certain arrangement may prove ineffectual when compared to other arrangements. So, to study the relative performance, one must analyse all possible structures or predict by extrapolating a common trend influenced by the physical factors. 